\title{
Electricity, Gas and Other Fuels as Heating Agents
}

$\mathrm{I}^{\mathrm{N}}$ a paper read by Mr. A. H. Barker to the Institution of Electrical Engineers on December 1, an explanation was given of the conditions under which electrical power can be used competitively for the heating of buildings. It is well known that, reckoned on the heat unit basis alone, electricity is the most expensive of all the sources of heat in common use, and that comparatively, gas is at present not very far behind it. Since the heat in a gas company's therm equals $29 \cdot 4$ electric units, it follows that if gas cost $6 d$. per therm and electricity $0.5 d$. per unit, the cost of the crude heat per therm delivered by the gas company would be $6 d$. and by the electric company $14 \cdot 7 d$. In a few years' time we hope that more companies will be supplying at these cheap rates. If the thermal electric storage were employed the price of the electric heat would be reduced by about forty per cent. With oil at $80 \mathrm{~s}$, a ton and coke at $40 \mathrm{~s}$. a ton, the costs would be $2 \cdot 25 d$. and $1 \cdot 64 d$. a therm respectively. Looking merely at the costs of the 'crude' heat, the solid fuels are much cheaper.

The most fundamental difference between gas and electric heat is that with the former it is purified fuel which is transported to the spot where the heat is required, while with the latter it is energy only. There are three points arising from this. The street main is much larger and more difficult to accommodate with gas than with electricity but the housepipes for gas are smaller and cheaper. In order to convert the gas energy into heat, it has to undergo the process of combustion with the disadvantages of high temperature and waste products of combustion. The use of gas causes less actual destruction of fuel than electricity and the heat from it is therefore cheaper. Gas is in fact crude fuel which has had all the ashes and smoke purified out of it at the sacrifice of the manufacturing costs and about twenty per cent of the energy of the crude fuel. Electricity carries the purification a stage further. In its manufacture, everything, including all the labour, is purified out of the fuel but there is a loss of about twenty per cent of the energy in the mains. There are obviously cases, where so far as its usefulness is concerned, this further stage of purification is a pure waste of money, just as it is a waste of money to soften water needed for sanitary fittings.

In Mr. Barker's opinion, gas and electric supply have each their own sphere of usefulness, in which one is either economically or functionally superior to the other. There is only a small area of supply in which reasonable competition is legitimate. Both industries ought to be amalgamated, in their own and in the public interest. It is very difficult to assess the money value of their relative advantages and disadvantages in each particular case.

Apart from its cost, electrical energy is almost an ideal means for room warming. By its agency, pure heat can be delivered through light and flexible wires in any quantity, at any temperature and in any desired form to any particular spot. Turning on and off involves nothing but a mechanical motion of a switch and this can be readily done automatically by a simple and trustworthy form of thermostat. The use of any other fuel gives, along with the heat, products of combustion of a more or less deleterious nature. It is only in very special cases that heat derived from the combustion of fuels can be employed without the use of chimneys.

Gas possesses the advantage that it can be exactly regulated to requirements. It can be conveniently stored so that a sudden overload need not affect the supply. Breakdown is less probable than with the more complicated electrical plant. In some cases when combustion is effected completely, the products are innocuous and so can be allowed to mix with the air of the building and so securelike the electrical fire-an efficiency of one hundred per cent. The drawbacks are that gas needs to be ignited and supplied with air before the heat can be developed. It has an objectionable smell and is dangerous if it escapes or is incompletely burnt.

The advantages of oil are that it is fluid and so can be pumped with little smell or trouble into a tank through a pipe-line. It is much easier to ignite, to regulate and to extinguish than coke, though more difficult than gas. It is very clean in operation, and when properly burnt highly efficient. Owing to the high degree of the combustibility of oil, there is a good deal of potential danger attached to it. It is apt also to give off smoke and odorous fumes. The advantages and disadvantages of coke and other solid fuels are well known. For example, they will allow any sort of combustible material to be destroyed in the furnace. It is probably the most economical form of heating but it involves greater labour than any other fuel. It produces dust, is dirty to deliver and clumsy to handle.

Mr. Barker deals exclusively with the cost of the fuel and the labour involved in handling it. When the heating required is intermittent, both electrical and gas heating have advantages over the other fuels.

\section{Mesolithic Age in Britain*}

$\mathrm{I}^{\mathrm{N}}$ 1926 the Royal Anthropological Institute held an exhibition illustrating the microlithic industries of Britain, to which all who were then known to be interested and engaged in forming collections of implements of this phase of the Stone Age were asked to contribute. The mesolithic period had been somewhat neglected by British archæologists; and it is probable that it came as a surprise, even in archæological circles, to find how considerable was the amount of material which it had been possible

*Based on certain papers read before Section $H$ (Anthropology) of the British Association at York, September 6, 1932. to get together and the increase in the interest taken during the early years following the War in these remarkable products of man's skill and ingenuity.

In the period which has elapsed since that exhibition was held, further progress has been made in the study of the mesolithic age, and the time was fully ripe for a detailed discussion of the position of microlithic industries in relation to preceding and succeeding cultures. The opportunity for such discussion was afforded by a series of papers in the programme of Section $\mathrm{H}$ (Anthropology) when the 